\title{
La manipulación de los materiales como recurso didáctico en educación infantil
}

\author{
Francisco Manuel Moreno LuCAS \\ Universidad Católica San Antonio de Murcia \\ fmoreno@ucam.edu
}

Recibido: $26 / 10 / 2012$

Aceptado: 23/01/2013

\begin{abstract}
Resumen
Los materiales son elementos indispensables en educación infantil por el alto marco de influencia que ejercen estos en el proceso de enseñanza/aprendizaje en los niños y niñas. De esta manera el estudio y la investigación de los materiales y recursos que se utilizan en las primeras etapas educativas, como el conocimiento de todo los materiales y recursos disponibles, la realización de una buena clasificación y selección del material en el aula, contribuirá a un mejor aprendizaje y proporcionará una fuente educativa donde poder acudir para ofrecer a los infantes una educación de calidad y en vías a la excelencia.
\end{abstract}

Palabras clave: materiales, recursos, educación infantil y aprendizaje.

\section{The Manipulation of Materials as a Teaching Resource in Childhood Education}

\begin{abstract}
The materials are essential elements in early childhood education because of their high influence in the teachings / learnings for children, so that way the study and research of materials and resources used in the early stages of education, like knowledge of all the materials and resources, the making of a good classification and the selection of the material in the classroom will all contribute to a better learning, and provide an educational resource where we could turn for offering a quality education to the infants and directed for excellence.
\end{abstract}

Keywords: materials, resources, early childhood education and learning.

\section{Referencia normalizada}

MORENO LUCAS, Francisco Manuel (2013): "La manipulación de los materiales como recurso didáctico en educación infantil". Estudios sobre el Mensaje Periodístico. Vol. 19, Núm. especial marzo, págs.: 329-337. Madrid, Servicio de Publicaciones de la Universidad Complutense.

Sumario: 1. Introducción. 2. Metodología. 3. Desarrollo. 4. Conclusiones. 5. Referencias bibliográficas

\section{Introducción}

En educación infantil los materiales tienen una gran relevancia por ser elementos de primer orden en el proceso de enseñanza de los alumnos, de esta manera, es importante la clasificación, selección y utilización de los mimos como indicadores notables sobre la calidad y eficacia de la formación.

En educación cualquier material puede utilizarse como un recurso en el proceso de enseñanza/aprendizaje, pero no todo material se concibe para dicho fin. Multitud de materiales son utilizados en educación infantil con fines educativos, al igual que existe un extenso catálogo de materiales que se utilizan con carácter educativo, aunque la finalidad para la cual fueron creados no fuera pedagógica. Gracias a la creatividad, imaginación, investigación y experiencia de los docentes, estos materiales se adaptan a las necesidades y características de los alumnos con el fin de alcanzar las competencias educativas perseguidas. 
Cuando hablamos de materiales y recursos que se utilizan en educación, estamos abarcando a un gran abanico de elementos, enseres, herramientas con las cuales interactúa los educandos, es decir, todo lo que condiciona e interacciona con ellos lo podemos considerar material, como el mobiliario, los juguetes, el material didáctico, materiales informáticos y tecnológicos, así como cualquier otro elemento que implique una acción del niño y contribuya a un aprendizaje.

Autores como Doménech y Viñas (1997), consideran que en el desarrollo educativo de los alumnos de educación infantil, juegan un papel muy importante los materiales que utilizamos en el proceso de enseñanza/aprendizaje, siendo éstos, elementos mediadores entre el educador y el entorno que lo rodea.

A la hora de apostar por una educación infantil de calidad, como apunta Peña (2003), tenemos que trabajar en el desarrollo de las dimensiones físico, afectivo, cognitivo y social, y para ello es primordial la planificación de los diversos recursos y materiales que vamos a utilizar para que el aprendizaje de los discentes sea verdaderamente significativo.

Ameijeiras define los materiales en educación como "todo el amplio campo de los objetos que se ponen a disposición del niño" (2008: 93), de esta manera, todo los elementos que rodean al niño tienen como primera función la de ser elemento mediador entre su conocimiento y la realidad que lo rodea, es como la puerta a través del cual el niño empezara a descubrir, a explorar, todo su entorno.

Siguiendo las aportación que realiza Rodríguez Cancio (2005: 55) "el principio que debe regir en la utilización del material es el uso vivo e inteligente de las cosas", poniendo de manifiesto que un material por sí solo no puede educar, no posee la cualidad de apoyar la formación, sino que necesita la implicación e intervención del educador para conferirle el significado propio perseguido a través del aprendizaje. Un material es educativo en función del significado que el docente le transfiera.

Martínez Sánchez (1993: 241) considera material didáctico como el "formado tanto por objetos de uso cotidiano y familiar como por recursos elaborados específicamente para la escuela". A la hora de la planificación del proceso de enseñanza/aprendizaje, tiene una primordial influencia la selección y utilización que hagamos de todos los materiales que dispongamos para la formación de los alumnos.

Marqués Graells (2000) detecta que los materiales pueden clasificarse, sobre todo, en dos tipos según el fin por el cual han sido elaborado, de esta manera nos encontramos con los medios didácticos, que engloban a todo tipo de producción o material elaborado con una finalidad educativa; y los recursos educativos, donde se aglutina a todo ese material cuya confección no ha sido ideada para el ámbito educativo, pero si se utiliza con fines didácticos. De esta manera consideraremos material educativo, tanto a todo elemento que ha sido creado con un fin educativo como a todo elemento que cumpla un fin pedagógico, aunque en un principio haya sido confeccionado para otros fines.

Los recursos materiales son todos los elementos que podemos utilizar para el proceso de enseñanza/aprendizaje de los alumnos, desde el punto de vista de componente activo del aprendizaje y como herramienta que contribuye y facilita la consecución de los fines educativos. 
Los medios materiales, según Rodríguez Cancio (2005), son elementos favorecedores de los aprendizajes, sin tener ninguna función propia por sí mismo, ayudan de forma eficaz y eficiente como soporte esencial para relacionar los aprendizajes con las metodologías y actividades que se desarrollan para conseguir los objetivos planteados. Se puede decir, que los materiales son un soporte que tienen la finalidad de respaldar la labor educativa, son el nexo de unión entre el aprendizaje y la metodología utilizada.

\section{Metodología}

Autores como Abengoechea y Romero (1991), hablan de la función innovadora de los materiales, como productora de estímulos ante lo novedoso y creativo que aporta el material al alumno, y la función condicionadora del aprendizaje, en relación a la clase de procesamiento de la información que los alumnos realizan a través de las impresiones de los materiales.

Doménech y Viñas (1997), están de acuerdo en que la función básica de los materiales es la de complementar y servir de base a la actividad educativa que se desarrolla en cualquier espacio educativo. La función innovadora y motivadora ayudara en el itinerario educativo de la adquisición de los nuevos conocimientos.

Bautista (2010), apunta sobre la función de los materiales como base y complemento en la actividad educadora, por tener éstos varias características que contribuyen a ello, como:

- Carácter motivador; ya que según la forma, textura, color y características particulares del material, despertara el interés y curiosidad para su utilización.

- Carácter polivalente; los materiales pueden ser utilizados como elementos en varias actividades escolares y como elementos de diferentes juegos.

- Carácter colectividad; su utilización puede ser individual o colectiva.

- Carácter de accesibilidad; estar organizado y disponible para la libre elección de los alumnos.

Martínez Sánchez (1993), presenta como importante el que el profesor seleccione y clasifique los materiales según los fines que se persiguen, así como los ritmos y necesidades de los alumnos. Debe de presentarse al alumnos diferentes materiales que puedan utilizar y contribuir al desarrollo en varios ámbitos, como son materiales que favorezcan el desarrollo de la comunicación y el lenguaje, materiales que fomente la educación sensorial, objetos que permita a los alumnos en contacto directo con la naturaleza y la exploración del entorno que lo rodea, etc.

La selección y la organización de los materiales ha de ser cuidadosa y consensuada, ya que serán estos recursos los que garantizaran el aprendizaje, reforzando la adquisición de la autonomía e independencia, y la interiorización de valores, normas y actitudes que ayuden a la socialización y relación entre iguales.

Son muchos los criterios a tener en cuenta a la hora de seleccionar el material que va a favorecer la adquisición de las competencias educativas, para ello resaltamos los criterios que Rodríguez Cancio (2005), hace de los materiales en educación infantil:

- El material tiene que ser atractivo tanto estéticamente como funcionalmente, tiene que llamar la atención del infante, invitándolo a interactuar con él. 
- Los materiales deben de ser adecuados al momento evolutivo del niño, adaptados a sus aptitudes, características y necesidades.

- Que sean asequibles y manipulables, que los niños puedan utilizar el material de forma autónoma e independiente.

- Que potencie y favorezca la actividad motora, cognitiva, afectiva y social.

- Que reúna las correspondientes normas de seguridad.

- Que proporciones los estímulos correspondientes a su elección.

- Que no favorezca actitudes de discriminación de sexo ni belicistas.

- Que posibilite al niño todas las posibilidades de acción que éste pueda desarrollar.

La Educación Infantil contribuye a desarrollar en los niños una serie de capacidades que les permitan conocer su propio cuerpo y sus posibilidades de acción; aprender a respetar las diferencias; relacionarse con los demás, a través de la afectividad, la convivencia y la relación social, la resolución pacífica de conflictos; observar y explorar su entorno natural, familiar y social; y adquirir autonomía, en todas sus actividades habituales.

Una clasificación que da respuesta a las diferentes capacidades y competencias que tiene que tiene que adquirir el alumno durante su recorrido por el segundo ciclo de educación infantil, es la siguiente:

- Materiales de manipulación, observación y experimentación; arena, agua, barcos, coches, botes y cajas de plásticos y cartón, tubos, juegos de medida, objetos de la naturaleza como semillas, huesos de frutas, conchas, piñas, plantas.

- Materiales que desarrollan el pensamiento lógico; enseres que permitan comprar, asociar, ordenar, clasificar, seriar, contar, medir, como juguetes, ropa, comida, carretes de hilo, lanas, cajas o palos con diferentes tamaños, colores, texturas y medidas de capacidad, dominó, cartas, bloques lógicos, juego de mesa, ábacos, dinero de mentira, cinta métrica.

- Materiales para representación y simulación; sirven para iniciar su conocimiento del mundo, ya que el niño necesita imitar aquellas situaciones de la vida del adulto a través de la simbolización. Importante el guiñol o teatro de títeres.

- Materiales no estructurados; botones, palos, bolsas, cuerdas.

- Materiales recogidos de la comunidad; vajillas, objetos de cocina, maquillaje, bisutería, ropa y zapatos de adulto, bolsas, carteras, ropas de bebé, peine, cepillos, sombreros.

- Materiales comprados; mercados, casitas de muñecas, maletín medico, de carpintero, teléfono, máquina de fotos.

- Material para el desarrollo de la expresión oral; sirven para la adquisición básica del lenguaje, que pueden servir todo el material descrito anteriormente, pero también tenemos que hacer mención a :

- Colecciones de imágenes; laminas murales para hacer preguntas en relación a la imaginación sensorial, visual (colores, formas), auditiva (ruidos, ej. ¿qué hacen los perros?), gustativa (dulce, salado), táctil (cosas áspera, suaves, frías), y olfativas. Relaciones lógicas, cosas que hay en casa, en el campo, cosas que 
hay en todas las láminas, arriba o abajo. Situación del tiempo (día, noche, verano), de causa o efecto (porque llevan paraguas, porque esta subido a la escalera), de numero, cantidad, volumen.

- La grabadora; muy útil para el lenguaje, realizando dos actividades básicas, escuchar y grabar.

- Libros para ver y contar.

- Materiales para la expresión plástica y musical; están relacionadas con el lenguaje y apoyan la comunicación y expresión oral. También cumplen con el desarrollo de la habilidad manual, donde es más importante el proceso que el resultado.

De esta manera, todos los objetivos que se deben de conseguir en educación infantil, pueden ser adquiridos a través de la utilización y mediación de multitud de materiales, cuya función, a parte de la de contribuir a la adquisición de aprendizajes, es hacer más fructífera y fácil, la adquisición de las competencias tan necesarias para su posterior formación en etapas superiores.

\section{Desarrollo}

La mayoría de los aprendizajes que se realizan en educación infantil, es a través de la experimentación, la manipulación, la utilización de recursos materiales que provoquen la estimulación de todos los sentidos. Principalmente en los primeros años de vida, los sentidos más propicios para la adquisición del aprendizaje serán el táctil y el visual, sin dejar de lado los demás, pero el impulso hacia el descubrimiento, la curiosidad, el deseo de saber y conocer el entorno inmediato, contribuirá a que el niño utilice principalmente éstos.

Soler Fiérrez (1993), comenta que a través de los sentidos, los niños van construyendo sus propias ideas en relación al mundo en el que vive, mediante la experimentación táctil va explorando su entorno, conociendo el mundo que lo rodea, todo circula mediante un mecanismo de ver los objetos, manipularlo y explorarlo a través del gusto, por este motivo, es habitual ver a los niños más pequeños llevarse a la boca todo lo que encuentra a su alrededor.

La gran pedagoga Montessori (1937, citado en García, 1993), daba mucha importancia a la educación pedagógica de los sentidos, a partir de éstos, los alumno obtendrá mas información y podrán con ello sintetizar y adquirir los nuevos aprendizajes. En educación infantil, los sentidos que más se trabajan son el tacto, la vista y el oído, siendo éstos, canales más cómodos para trabajar los aprendizajes.

El aprendizaje a través de la manipulación de los múltiples materiales que podemos encontrarnos en el aula, se da precozmente y es primordial, como apunta Boulch (1983), para el descubrimiento de la realidad operante y la adquisición de nociones espaciales y temporales.

A la hora de que el alumno adquiera las experiencias, destrezas, habilidades que le ayudaran a su desarrollo global, es necesario como apunta Martínez Sánchez (1993), la manipulación, el manejo y empleo de diferentes recursos y materiales didácticos con el objetivo de que éstos faciliten la adquisición de los aprendizajes y conocimientos 
deseados. La utilización de los recursos materiales contribuirá como estimulo para el aprendizaje en el área psicomotor, en el proceso de socialización, en la educación sensorial y sobre todo en el área manipulativa.

Bruner, J.S. et al (1977), apunta sobre el carácter atractivo que tiene que tener el material para su posterior manipulación, ya que la experimentación manipulativa que realiza el discente, conlleva la adquisición de capacidades cognitivas, de interacción y socialización.

La utilización de una variada selección de materiales manipulativos ejercerá una gran influencia en la manera y la evolución de los aprendizajes, tal y como apunta Campos Villalobos:

"A palpar los objetos materiales: reconoce Froebel que el tacto es otro medio perceptivo capaz de entregar conocimiento al niño y se aconseja que aprenda, por ejemplo, a reconocer las formas geométricas por el tacto y no solamente por la vista. También señala que en un orden evolutivo de desarrollo infantil, es el medio que más temprano aparece" (Campos Villalobos (2011: 1)

Con los jardines de infancia, Froebel ya consideraba la exploración, la experimentación, la manipulación a través del tacto como una parte importante en el proceso de desarrollo de los alumnos, ya que a través del trabajo manual, en contacto directo con los elementos y materiales cotidianos que rodean al niño, se favorece la adquisición de aprendizajes en todos los niveles, cognitivo, afectivo y social.

Una de los primeros estímulos que provocan en los niños el deseo de manipulación, es la curiosidad, y tal como indica García Hoz (1993), será a través del manoseo, el inicio de los primeros avances en el niño en su afán de conocer el mundo que lo rodea. Esta tendencia a tocarlo todo, se irá intensificando conforme va creciendo, y será una de las principales vías de aprendizaje que tendrá el niño para su pleno desarrollo madurativo.

Bautista (2010), habla sobre la importancia de presentar a los alumnos diferentes materiales, para que tras su manipulación y experimentación provoque estímulos para el desarrollo de aptitudes lingüísticas, motrices, emocionales y psicológicas.

Los niños tienen que manosear, tocar, tantear, palpar, de manera física los objetos, para poder aprender a partir de ellos. En esta línea Heiland (1982), afirma que la educación preescolar está justificada por lo imprescindible que la educación sensorial se hace presente en esta etapa, ya que los niños que pasan del primer ciclo de educación infantil, habiendo recibió una pedagogía abundante en lo sensorial, tiene que seguir recibiéndola, mayoritariamente en el primer curso del segundo ciclo de educación infantil.

Good y Brophy (1980) afirma que es necesario tener en cuenta el nivel de desarrollo de cada niño para determinar los objetivos que se quieren conseguir, para que a partir de la experimentación y manipulación de los materiales adecuados, favorezcan su aprendizaje y su desarrollo.

Los materiales constituyen un instrumento muy importante para el desarrollo del niño dentro de la tarea educativa, ya que toda su actividad, juegos y aprendizajes la realizan a través de juguetes, objetos, instrumentos, etc. Es a través de la manipula- 
ción con los objetos, explorando sus características y funciones, donde dan rienda suelta a su imaginación y a su aprendizaje. Soler Fiérrez (1993) orienta sobre una serie de actividades que tendrían que realizar el alumnado de infantil con el fin de conseguir los objetivos marcados en el curriculum, entre todos los objetivos que menciona resalto los siguientes:

- Comprobar los contrastes a través del tacto de distintos materiales como la arena, el agua, distintos tipos de legumbres, piedras, serrín, etc.

- Transportar los objetos de un lugar a otro con diferentes recipientes como bolsas, cajas, cubos, etc.

- Reconocer a través de la manipulación diferentes enseres de la vida cotidiana, como cuchara, recipientes tetrabrik, pelota, libros, herramientas, etc.

\section{Conclusiones}

La utilización de los materiales en educación infantil es importante para el posterior desarrollo a nivel global de los discentes de esta etapa. Es fundamental, la realización de una buena planificación y la confección de una adecuada selección de materiales con el fin de adaptarnos a las características individuales de cada alumno.

Siguiendo a Moreno (2011), la educación es un proceso que se desarrolla durante toda la vida, y tenemos que comenzar con una enseñanza/aprendizaje desde los primeros años de vida, a través de la interacción con elementos materiales que estimulan a la acción, el manoseo, aproximando a los niños actividades para que desarrollen los sentidos como la vista, el tacto, el gusto el oído, en definitiva, utilizar una metodología donde puedan desarrollar todos sus sentidos para conocer, investigar, aprender. Disponer de una buena fuente de recursos materiales, como estrategias, pedagogías, metodologías, donde poder recurrir, convertirá en nuestra enseñanza en un proceso más personalizado y adaptado a las necesidades e intereses de nuestros educando.

La enseñanza por medio de la manipulación de materiales y recursos en educación infantil, es una metodología de aprendizaje que nos acerca más a la idiosincrasia de los alumnos, así como un recurso potencial donde poder trabajar todas las competencias educativas que tendrán que interiorizar en años posteriores.

\section{Referencias bibliográficas}

ABENGOECHEA, S. y ROMERO, E. (1991): Selección y empleo de medios. Máster de formación de formadores. Barcelona, RENFE-UAB (policopiado).

AMEIJEIRAS SAIZ, Rosa (2008): Manual de Educación Infantil. Aspectos didácticos y organizativos. Cáceres, Universidad de Extremadura.

ALBERT GOMEZ, María José (2009): La investigación educativa: claves teórica. Madrid, McGraw-Hill.

AREA MOREIRA, Manuel (2004): Los medios y las tecnologías en la educación. Madrid, Pirámide.

ANTÓN ROSERA, Montserrat (coord.) (2005): Planificar la etapa 0-6años. Compromiso de sus agentes y práctica cotidiana. Barcelona, Grao. 
ANTON ROSERA, Montserrat y MOLL FERRE. Blanca (coord.) (2000): Educación infantil, orientaciones y recursos ( 0 - 6 años). Barcelona, CissPraxis.

BAUTISTA VALLEJO, Jose Manuel (2010): "Los materiales como mediadores", en www.investigalog.com/el_juego_como_metodo_didactico/tema-8-los-materialescomo-mediadores/. [fecha consulta: 27 de junio de 2012]

BLÁZQUEZ ENTONADO, Florentino (1993): "El espacio y el tiempo en los centros educativos", en LORENZO DELGADO, Manuel \& SAENZ BARRIO, Oscar (Dir): Organización escolar, una perspectiva ecológica. Alcoy, Marfil, pp.339-365

BLUMER BERKELEY, Herbert (1982): El interaccionismo simbólico: perspectiva y método. Barcelona, Hora.

BRUNER, Jerome Seymour; JOLLY, Alison y SYLVA, Kathy (1977): Play: Its role in development and evolution. Harmondsworth, Penguin.

CAMPOS VILLALOBOS, NELSON (2011): "Froebel: Un pedagogo comprometido", en http://filo-edu.blogspot.com.es/2007/12/froebel-un-pedagogo-comprometido.html. [fecha de consulta: 17 de mayo de 2012].

CUELLAR PEREZ, Hortensia (2005): Froebel. La educación del hombre. México, Trillas.

DEL HERRÁN GASCON, Agustín \& PAREDES LABRA, Joaquín (2008): Didáctica general. La práctica de la enseñanza en Educación Infantil, Primaria y Secundaria. Madrid, McGraw-Hill.

DOMÉNECH, Joan y VIÑAS, Jesús (1997): La organización del espacio y del tiempo en el centro educativo. Barcelona, Grao.

GARCÍA HOZ, Víctor (Dir) (1993): Educación infantil personalizada. Madrid, Ediciones Rialp.

GOLDSCHMIED, Elinor y JACKSON, Sonia (2007): La educación infantil de 0 a 3 años. Madrid, Ediciones Morata, S.1.

De PABLO, Paloma y TRUEBA, Beatriz (1994): Espacios y recursos para ti, para mí, para todos. Diseñar ambientes en educación infantil. Madrid, Escuela Española.

GERVILLA CASTILLO, María Angeles (1994): Educación infantil: proyecto de investigación y bibliografía básica. Málaga, Servicios de Publicaciones de la Universidad de Málaga.

GOOD, T.H.L y BROPHY, J.E. (1980): Educational Psichology. Nueva York, Holt, Rinegart and Winston.

LE BOULCH, Jean (1983): El desarrollo desde el nacimiento a los seis años. Madrid, Ed. Doñate.

MARTÍNEZ SANCHEZ, Amparo (1993): “El aula en la educación infantil”, en GARCÍA HOZ, Víctor (Dir.): Educación infantil personalizada. Madrid, Ediciones Rialp, pp. 225-261

MORENO LUCAS, Francisco Manuel (2011): "Proyecto de innovación educativa sobre la formación para las personas que acceden a la prueba de acceso a la Uni- 
versidad para mayores de 25 años" en Revista Vivat Academia, año XIII, $\mathrm{n}^{\circ} 116$ : http://www.ucm.es/info/vivataca/numeros/n116/DATOSS.htm\#pro

MUÑOZ PEINADO, Jesús (2004): Enseñanza-aprendizaje en estrategias metacognitivas en niños de educación infantil. Burgos, Universidad de Burgos.

ORDEN ECI/3960/2007, de 19 de diciembre, por la que se establece el currículo y se regula la ordenación de la educación infantil.

PEÑA ACUÑA, Beatriz (2003): "Reflexiones acerca la enseñanza e la sociedad actual". Revista de la SEECI, ${ }^{\circ} 10$. Madrid, Universidad Complutense de Madrid. Facultad de Ciencias de la Información, pp. 53-74.

POVEDA DE AGUSTIN, Jose María (1981): Locura y creatividad. Madrid, Alhambra.

REAL DECRETO 1630/2006, de 29 de diciembre, por el que se establecen las enseñanzas mínimas del segundo ciclo de Educación Infantil.

RODRÍGUEZ CANCIO, Mónica (2005): Materiales y Recursos en educación infantil. Manual de usos prácticos para el docente. Vigo, Ideaspropias Editorial.

SOLER FIERREZ Eduardo (1993): "La educación sensorial: fundamentación, panorama, metodología y objetivos", en GARCÍA HOZ, Víctor (Dir.): Educación infantil personalizada. Madrid, Ediciones Rialp, pp.195-224

VIDORRETA GARCIA, Concha (1982): Como organizar un centro de recursos. Madrid, Anaya.

VYGOTSKY, Lev Semiónovich. (1979): El desarrollo de los procesos psicológicos superiores. Barcelona, Critica.

ZABALZA BERAZA, Miguel Ángel (2008): Didáctica de la educación infantil. Madrid, Narcea. S.A. de Ediciones.

\section{Francisco Manuel MORENO LUCAS}

Universidad Católica San Antonio de Murcia

Licenciado en Pedagogía

Profesor docente investigador

fmoreno@ucam.edu 